\title{
EVOLUTIONARY REDUCTION OF ENZYMATIC ACTIVITIES OF SNAKE VENOM PHOSPHOLIPASES $\mathrm{A}_{2}$
}

\author{
INN-HO TSAI \\ Institute of Biological Chemistry, Academia Sinica, and Institute of Biochemical \\ Sciences, National Taiwan University, Taipei, Taiwan, Republic of China
}

\begin{abstract}
The reaction mechanism of the $14 \mathrm{kDa}$ secreted phospholipases $A_{2}$ (PLAs) and examples of venom PLAs with diminished catalytic activities are reviewed. Evolutionary strategies to reduce the venom PLA catalytic power and new function gains are discussed. Down-regulations of the enzymatic activities appear to be due to: 1) retention of interfacial binding, but with selective alternation of active site residues in basic PLAs; 2) mutations at both the interfacial binding sites and catalytic sites of strong anticoagulating PLAs which bind to the coagulation factor; and 3) either substitution or truncation of the interface binding sites in acidic subunits of heterodimeric PLA-neurotoxins to generate chaperon like molecules.
\end{abstract}

Keywords: Phospholipase A2, Snake venom toxin, Interface binding, Catalytic site, Oxyanion hole, Regulation

\section{Introduction}

Phospholipases $\mathrm{A}_{2}$ (PLAs) are major components in snake venoms and usually exist in multiple isoforms or paralogs. These relatively small (14 kDa, EC3.1.1.4) secreted hydrolases are calcium dependent enzymes, which hydrolyze the $s n$-2 ester of glycerophospholipid to produce a fatty acid and a lysophospholipid (Six and Dennis, 2000; Gubensek and Kordis, 1997). The venom PLAs have been classified according to minor structural differences as group IA, IB, IIA and IIB. The former two are present in elapid and hydrophiid venoms while the latter two are present in viperid venoms. Up to 300 venom PLA 2 s have been so far sequenced (Kini et al., 1997; Danse et al., 1997). They show $>50 \%$ sequence identity and have a conserved 3D-protein

Address correspondence to Inn-Ho Tsai, P.O. Box 23-106, Taipei, Taiwan 10798. E-mail: bc201@gate.sinica.edu.tw 
scaffold (Scott, 1997), but their catalytic activities differ greatly and are not necessarily related to the toxicity (Rosenberg, 1997). The venom PLA families induce a wide spectrum of toxic or pharmacological effects, and some possibly play more than one roles. Thus, their structure-function relationships are subtle and intriguing challenges.

The PLAs gain their full activity only when they bind to phospholipid micelles or membranes, an effect known as interfacial activation (Gelb et al., 1995; Qin et al., 2005). X-ray structures are available for more than 20 of the venom PLAs, both in free form and as complex with catalytic site inhibitors (Kini, 2005; White et al., 1990; Ward et al., 1998; Pan et al., 2002; Murakami et al., 2006). NMR and other spectroscopic studies identified conformational changes in the PLAs upon binding to phospholipid micelles or membranes, i.e. the first few residues of the N-terminus are flexible in solution, but upon micelle binding its $\alpha$-amino group is engaged in H-bonding to the active site (Vandenberg, 1995a,b). Membrane potential may also affect PLA enzymatic activity (Thuren et al., 1987; Ward et al., 1998). It was shown that membrane binding of group IIA PLAs is accompanied with destabilization of the N-terminal $\alpha$-helices, while the $\alpha$ helices of the group IB PLAs adopted more fixed conformation upon binding the phospholipid micelles (Qin et al., 2005).

The collar on the surface of the PLA molecule, that surrounds the slot entrance of monomeric substrate (i.e., residues $3,6,7,19,20,31,64-69,113,116,122)$, appears to function as the interface binding surface (White et al., 1990; Koduri et al., 1997; Scott, 1997; Qin et al., 2005). Results from study by Fourier transform infrared spectroscopy (FT-IR) (Qin et al., 2005) suggested that the enzyme probably forms a water tight seal with the interface, and the phospholipid must travel about $7 \AA$ into the catalytic site slot to reach the catalytic network. The substrate's acyl chains contact hydrophobic amino acids including residues 4, 5, and 9 that line the walls of the slot (White et al., 1990; Gelb et al., 1995; Pan et al., 2002; Qin et al., 2005). The conserved catalytic site residues of PLAs have been probed by mutagenesis and 3D structural studies (Thunnissen et al., 1992; Gelb et al., 1995); an aspartate-histidine-water catalytic triad was identified, that is analogous to the triad found in serine proteases except that the serine is replaced by water molecules hydrogen bonded 
to the imidazole ring of His48. It was proposed that water is the attacking nucleophile and the tetrahedral intermediate formed at the substrate's $s n$ - 2 ester in the transition state is stabilized by an "oxyanion hole" near the main chain amide group of Gly30. The active site $\mathrm{Ca}^{+2}$ ion is coordinated by Asp49 and backbone carbonyl groups from a Gly-rich binding loop at positions 26-32 of the PLA.

The present review focuses on natural structural alternations of venom PLAs, which lead to toxins with low catalytic activities. Major examples of catalytically inactive variants of PLA during venoms evolution of elapid or viperid species will be discussed in categories.

\section{Materials and Methods}

\section{Venoms and Other Materials}

Bungarus fasciatus venom (Thailand origin) was purchased from Miami Serpentarium Labs. Pseudechis australis and Pseudechis porphyriacus were purchased from Australian Reptile Park (Gosford, New South Wales, Australia). Synthetic L-dipalmitoyl glycerophosphocholine was purchased from Avanti Polar Lipids (Alabaster, AL). Other chemicals were from Merck, Aldrich or Sigma.

\section{Purification of Venom PLA}

The venom PLAs of B. fasciatus were purified using the methods of Liu et al. (1988, 1989, 1990, 1994). After repeated centrifugations at 12,000 $\mathrm{g}$ for $5 \mathrm{~min}$, aliquots of $100 \mu \mathrm{l}$ dissolved venom $(15 \mathrm{mg})$ were injected into a gel-filtration column (Superdex75, HR10/30) on a Fast protein liquid chromatography system. The proteins were eluted in $0.1 M$ ammonium acetate $(\mathrm{pH}$ 6.4) at room temperature. Pseudexin A and B from $P$. porphyriacus, and PLAs Pa-11 and Pa-13 from $P$. australis were purified according to the published methods (Nishida et al., 1985; Schmidt and Middlebrook, 1989; Tabasaki et al., 1990). The PLAs were further purified by highperformance liquid chromatography (HPLC) on a RP-C18 column (Wang et al., 1999, 2005; Tsai et al., 2001). Fractions 
containing PLA activities were separately collected and freezedried in a vacuum centrifuge device (Labconco, CA).

\section{Enzymatic Activities and Kinetic Parameters}

The concentration of $\mathrm{PLA}_{2}$ in the stock solution was determined by the absorbance at $280 \mathrm{~nm}$, assuming an extinction coefficient of 1.5 at $1.0 \mathrm{mg} / \mathrm{ml}$ of the protein. Hydrolytic activities of PLA towards mixed micelles of L-dipalmitoyl phosphatidhlcholine and deoxycholate or Triton X-100 were assayed at $\mathrm{pH} 7.4$ and $37^{\circ} \mathrm{C}$ on a pH-stat apparatus (Radiometer, RTS 822, Denmark). The initial enzymatic rate was corrected for the spontaneous rate. Binding affinity of $\mathrm{Ca}^{+2}$ ion to the PLA was also determined by plots of $1 / V_{\max }$ versus $\mathrm{CaCl}_{2}$ concentration.

\section{Results and Discussion}

\section{Evolutionary Mechanisms of PLA Inactivation}

Normal reactions of PLAs include three steps. First, the enzyme binds to the interface and conveys a phospholipid molecule to the active site slot; second, the scissile ester bond of monomeric substrate is hydrolyzed by the catalytic triad with transition-state stabilization of the carbonyl oxyanion, and finally, the release of fatty acid and lysophospholipid product (White et al., 1990; Gelb et al., 1995; Pan et al., 2002; Qin et al., 2005). Thus, strategic inactivation of venom PLAs are usually related to any one or combination of the following mutations at: a) the structural motifs that recognize the interface; $b$ ) the structural features that control substrate binding; and c) the catalytic residues directly involved in the ester hydrolysis. I will discuss examples of natural PLA inactivation found in literature or recently studied by us in the following section.

\section{A. Reduced Catalysis with Retention of Interfacial Binding}

BASIC CATALYTICAlly inACTIVE G6/W6 PLAs OF CROTALid VENOM

Since D48 is normally the major side chain of PLA responsible for binding the active site $\mathrm{Ca}^{+2}$ ion, the PLAs with D49 $\mathrm{K}$ and Y28 $\mathrm{N}$ mutations can not bind $\mathrm{Ca}^{+2}$ at the active 
site, and thus lose hydrolytic activities (Lomante et al., 2003). The K49-PLA-homologs are the major form of venom PLAs of New World non-rattlers, e.g. Bothrops and Agkistrodon, and are apparently present in most venom species of Asian pit vipers, especially Protobothrops and Trimeresurus (Wang et al., 1999, 2005; Tsai et al., 2001). The venom contents are usually high, consistent with their noncatalytic roles. In general, this family of basic PLA homologs ( $\mathrm{pI}>9$ ) share $75 \%$ sequence identity. Furthermore, it was recently found that the K49R substitution occurred in this family of PLAs from Protobothrops (Wang et al., 1996; Tsai et al., 2004), and the major venom PLA of Malayan pitviper (Calloselasma rhodostoma) is an inactive PLA homolog with W6D49 substitution (Tsai et al., 2000). Besides biomembranes, these versatile toxins are capable of binding to polyanionic heparin or lipopolysaccharides. They also show strong myotoxic or cytotoxic and bactericidal activities, and may induce edema (Liu et al., 1991; Lee et al., 2001) and inflammation (Landucci et al., 2000; Zuliani et al., 2005). It has been shown that necrosis and apoptosis induced by them are associated with increments in cytosolic $\mathrm{Ca}^{2+}$ levels following plasma membrane perturbation, and possibly with the involvement of mitochondria (Mora et al., 2006).

In addition to position 49 , other alterations at the N-terminal region of the G6W6 basic proteins such as D4L5Q11 (which are normally Q4F5K11 in most crotalid D49-PLAs), probably impede the binding of phospholipid substrates to the hydrophobic wall of the K49-PLA family (Wang et al., 1996, 2005; Lomonte et al., 2003). Thus, the interfacial binding of K49-PLAs probably is not followed by entry of single substrates.

MYOTOXIC SER49 PLA IN VIPERINAE VENOM

Myotoxic PLAs are common components in viperid snake venom. Myotoxicity has been defined as the ability of proteins to induce skeletal muscle necrosis in vivo upon intramuscular injection, or in vitro upon incubation with differentiated skeletal muscle. Like the K49-PLA of pit viper venoms, G6S49-PLA homologs with impeded catalysis were found in the venom of some true vipers, especially African Echis (Polgar et al., 1996) and some European Vipera (Krizaj et al., 1991). Because binding of a single phospholipid molecule to the active site requires not only that the enzyme be bound to the interface, but also that the enzyme 
contain calcium, as shown by direct binding studies and inferred from X-ray structures, these group II PLAs without D49 may not bind substrate (Yu et al., 1993), and should have no direct catalytic activities.

PRO31-PLAS OF ELAPID VENOM

Most if not all elapid venom PLAs have a conserved D49 except that an inactive PLA with A49 (as well as N28D30) substitution was found in low content from the pooled venom of $B$. fasciatus (Liu et al., 1992). Instead, weakly catalytic or inactive forms of venom PLAs with a unique Pro31 residue are present in many elapid venom species, while other group I PLAs with higher catalytic activities have either Lys, Arg or Leu at position 31 (Liu and Lo, 1994). These P31-PLAs were found in the venom of Asian golden krait B. fasciatus (Liu and Lo, 1994; Liu et al., 1988, 1989, 1990), Australian Pseudechis (Nishida et al., 1985; Schmidt and Middlrbrook, 1989; Liu et al., 1990, Takasaki et al., 1990) and certain Hydrophiid species (e.g. Laticauda species) (Takasaki et al., 1988). Amino acid sequences of the basic P31-PLAs with low catalytic activities were aligned in Fig. 1. Many of them are abundant venom components, e.g. three P31-PLA isoforms (BfVa, BfVb-2 and BfVI) together comprised $45-55 \%$ of B. fasciatus venom protein content (Liu et al., 1990), and pseudexin B is about $15 \%$ of the protein in P. porphyriacus venom (Schmidt and Middlebrook, 1989). Notably, some of the nonenzymatic acidic subunits of highly potent taipoxin and texilotoxin in Australia elapid venoms (Fig. 1), and some inactive K49-PLAs of crotalid venom also contain P31 substitution (Tsai et al., 2001; Wang et al., 2005).

All these basic P31-PLAs have been reported to have no or very low enzyme activities toward various kind of aggregated and monodispersed substrates in vitro, but they may be more lethal than other PLA isoforms with higher catalytic activities. As shown in Table 1 the P31-PLAs Bf Va, Bf Vb-2 and Bf VI have much lower catalytic rate than $\mathrm{Bf}$ X-1 (containing K31), although all of them hydrolyzed phospholipid (lecithin) at $s n$ 2 ester position in a $\mathrm{Ca}^{+2}$-dependent reaction (Chang et al., 1983). The functions of the P31-PLAs have been reported to be like cobra cardiotoxins, myotoxins or membrane-acting cytotoxins, which cause membrane structure change or depolarization 


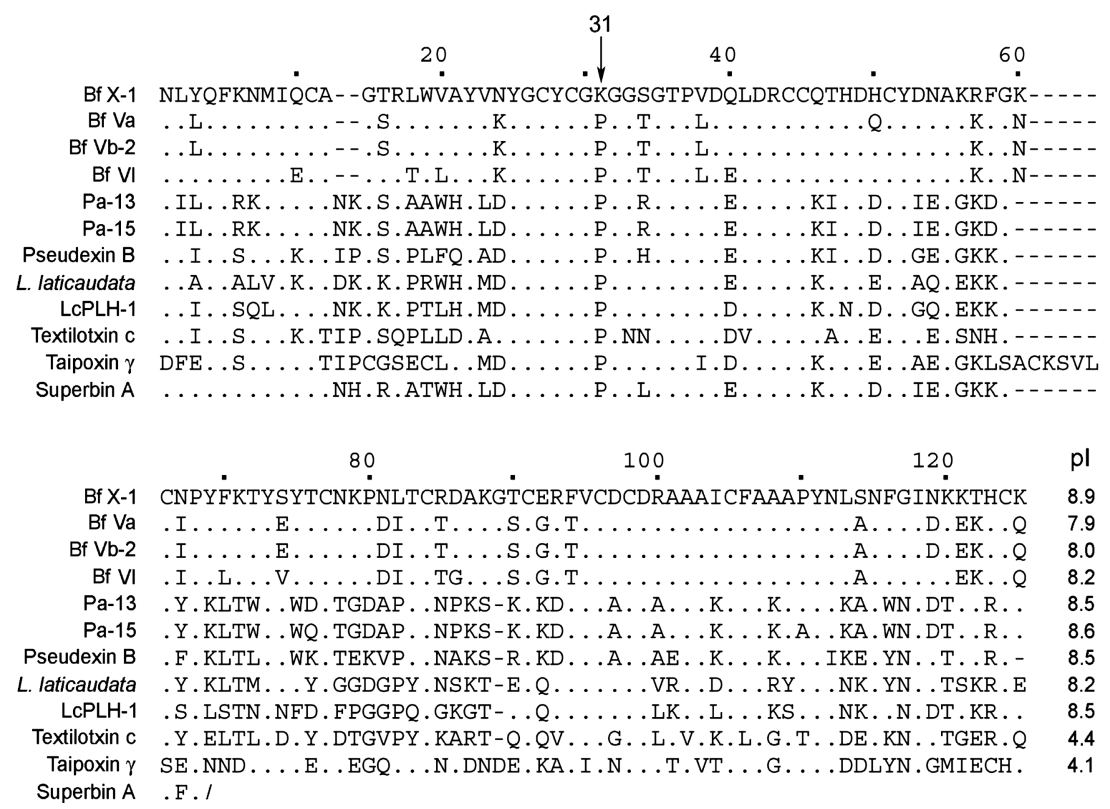

FIGURE 1 Sequence alignment of Elapid venom PLAs with Pro31 substitution. Single-letter codes of amino acids are used, and residues identical to those of a reference PLA, Bf X-1, are denoted with dots; gaps are marked with hyphens. Previous numbering system is adopted (Renetseder et al., 1985). GenBank or Swiss-prot accession numbers and the venom species for the PLAs are: Bungarus fasciatus: Bf X-1, P14615; Bf Va, P00628; Bf Vb-2, P00629; Bf VI, P00627; Pseudechis australis: Pa13, AAZ22665; Pa15, P20257; Pseudechis porphyriacus: Pseudexin B, P20259; Laticauda laticaudata: PLA-homolog, BAB72248; Laticauda colubrine: LcPLH-I, P10117; Oxyuranus scutellatus: Taipoxin $\gamma$, P00616; Pseudonaja textiles: Textilotoxin-C, AAZ22646; Austrelaps superbus: Superbin A, P59067.

(Shiaulin et al., 1975; Xu, 1986; Qi et al., 1989). It seems logical to speculate that the basic membrane toxins may be synergistic with other more active acidic PLAs (e.g., Bf X-1) to cause a severe cytotoxic effect or necrosis. Recently, a Tyr31-PLA without enzyme activity was isolated from Bungarus caeruleus, and the PLA existed as homodimer containing two mannose molecules H-binding to both Y31 and D49 (Singha et al., 2005).

Previous studies on pancreatic PLA mutants have revealed that replacements of L31 to other amino acids reduced the enzymatic activities considerably (Kuipen et al., 1990; Yu et al., 2000). Apparently, P31 in the $\mathrm{Ca}^{+2}$-binding loop of PLA could 
TABLE 1 Catalytic activities and lethal effects of B. fasciatus PLAs. Substrate used were: (a) micelles of $3 \mathrm{mM}$ dPPC plus $3 \mathrm{mM}$ sodium deoxycholate; (b) $3 \mathrm{mM}$ dPPC plus $6 \mathrm{mM}$ triton X-100; (c) micelles of egg lecithin and deoxycholate, with $5 \mathrm{mM} \mathrm{CaCl}_{2}$ and $0.1 \mathrm{M} \mathrm{NaCl}$ at $37^{\circ} \mathrm{C}$

\begin{tabular}{lccccc}
\hline \multirow{5}{*}{} & & \multicolumn{3}{c}{$\begin{array}{c}\text { Specific activity, mmc/min/g } \\
\text { enzyme }\end{array}$} \\
\cline { 3 - 5 } PLA & Residue & & & (i.p.) LD $_{50}$ \\
& 31 & (a) & (b) & (c) & $\mu$ g mice \\
\hline Bf Va & Pro & 10.2 & 38 & 0.4 & 4.7 \\
Bf Vb-2 & Pro & 5.9 & 27 & 1.6 & 4.7 \\
Bf VI & Pro & 5.8 & 10 & 0.3 & 3.6 \\
Bf X-1 & Lys & 50 & 320 & 42 & $>50$ \\
Bf III & Lys & 152 & 1000 & n.d. & $>50$ \\
\hline
\end{tabular}

affect the correct configuration of an oxyanion-hole involving backbone $\mathrm{NH}$ of Gly-30. In other esterases such as lipases, the contribution of oxyanion hole to the transition-state stabilization may be up to $20 \mathrm{~kJ} /$ mole, which account to an increase of catalytic rates by 100-1000 fold (Magnusson et al., 2005).

It is expected that the concentration of calcium required for maximal PLA activity will depend on the fraction of enzyme bound to the membrane and the affinity of the enzyme for the phospholipid substrate. In order to find whether the $\mathrm{Ca}^{+2}$ binding affinities or the turn over rate of P31-PLAs is inferior, we carried out kinetic analyses on these P31 PLAs. Our results showed that the P31-PLAs may bind $\mathrm{Ca}^{+2}$ strongly, with affinity constants of 13-49 $\mu M$ (Fig. 2) which are better than many other venom PLAs with higher catalytic power (affinity constants $>100 \mu \mathrm{M}$ ). The P31-PLAs prefer the zwitterionic micelles (Triton X-100) than the anionic micelles (deoxycholate) substrates. The $k_{\text {cat }}$ values of $\mathrm{Bf}$ VI calculated from double reciprocal plots are about 10-fold lower than that of Bf X-1 (a K31-PLA isoform), while the apparent $K_{\mathrm{M}}$ values of these two PLAs are less affected (Tsai, I. H., et al., 2007). It is very likely that the P31 substitution may disarrange or distort the structure of oxyanion hole essential for transition state stabilization, thus reducing $k_{\text {cat }}$ by about 10 -folds. 


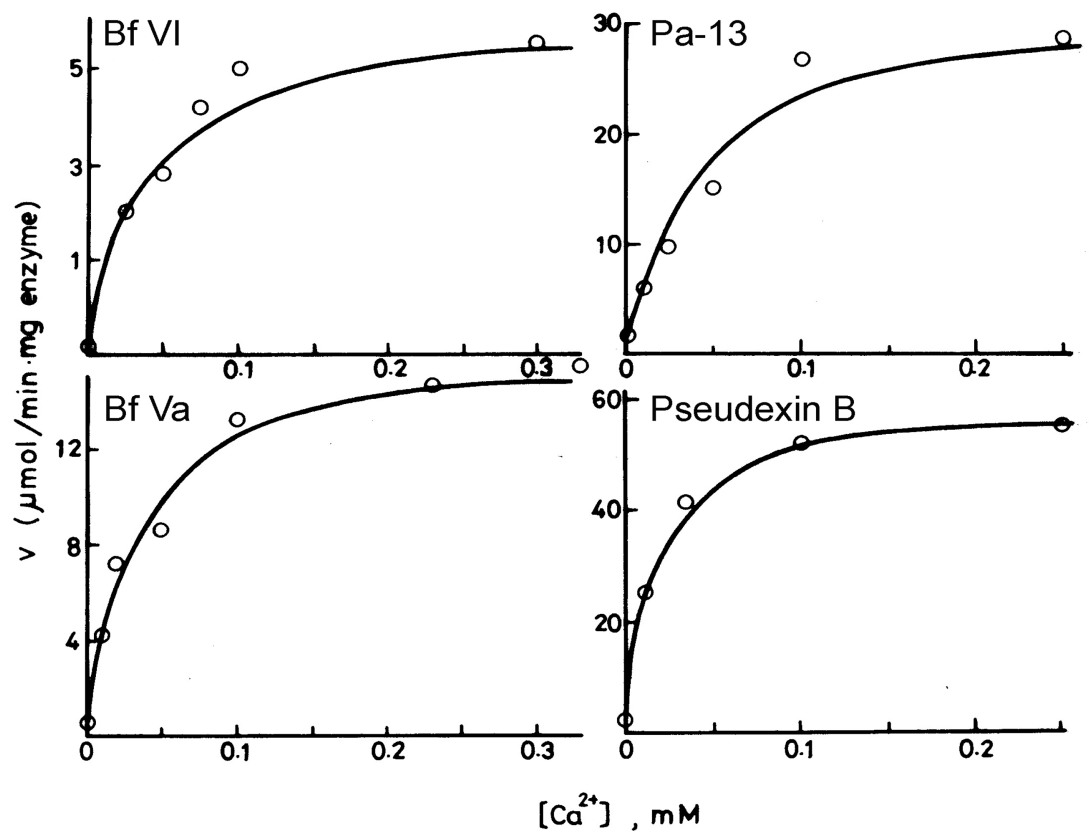

FIGURE 2 Dependence of hydrolysis rates of four venom P31-PLA on $\mathrm{Ca}^{+2}$ concentrations. The micellar substrates used was $3 \mathrm{~m} M L$-dipalmitoyl phosphatidylcholine and $6 \mathrm{~m} M$ triton $\mathrm{X}-100$, and initial rate of the enzymatic reactions were monitored at $\mathrm{pH} 7.2$ and $37^{\circ} \mathrm{C}$ on a $\mathrm{pH}$-stat apparatus in presence of $0.14-0.20 \mu M$ of the PLAs.

\section{B. Basic Anticoagulating PLA with Altered Interfacial Binding}

Anticoagulating PLAs are present in many genera of venomous snakes; their target mode and mechanism has been reviewed extensively (Kini, 2005). Anticoagulating effects of venom PLAs are often not related to the enzymatic activities (Condrea et al., 1982; Inada et al., 1994; Mounier et al., 2000; Zhong et al., 2002). Substitutions at both interfacial binding and catalysis sites appear to happen in the strong anticoagulating PLA-CMIV of Naja nigricollis venom (Kini, 2005). This group of PLA bear sequence similarity to factor $\mathrm{V}$ and tissue factor at region 54-71 (Kini, 2005; Jabeen et al., 2005), and may bind coagulation factor FXa in a hydrolysis independent mode and thus inhibit prothrombinase activation.

The residues of the i-face of PLAs are in an ensemble of conformations (Gelb et al., 1995), which apparently are effective 
in accommodating diverse binding targets such as coagulation factors. It appears that specific substitutions that may be unique to each PLA2, i.e. the N-and C-terminal part of human group IIA PLA, $\beta$-wing region and the C-terminal part of ammodytoxin (i.e., residues R72, K74, H76, R77) (Han et al., 1997), and residues E53, W70, T56 and D67 in basic venom PLA of Agkistrodon halys Pallas (Zhong et al., 2002), are responsible for their anticoagulating properties. In general, these substitutions altered interfacial binding sites (the i-face) of the PLA and enable it to bind coagulation factors.

Recently, new types of D49 substitutions to neutral amino acids (Asn and Gln) have been found in strong anticoagulating PLAs of many crotalid venom species. For example, R6N49PLAs were identified in the venom of Asian pit vipers including: Trimeresurus stejnegeri, Trimeresurus popeorum (Tsai et al., 2004), Trimeresurus albolabris (Rojnuckarin et al., 2006), Protobothrops mucrosquamatus (Wei et al., 2006) and possibly some other Protobothrops (Tsai et al., 2004). A PLA with special R6Q49 substitution was identified in the venom of Gloydius blomhoffii ussurensis (Bao et al., 2005). These PLAs thus cannot bind $\mathrm{Ca}^{+2}$ and are inactive enzymes. It is possible that $\mathrm{R} 6$ and basic residues at positions 7-15 also contribute to the lower catalytic activity of these anticoagulating PLAs since mutagenesis of E6R in an acidic PLA from Agkistrodon P. piscivorus resulted in lower $k_{\text {cat }}$ value (Prijatelj et al. 2006).

\section{Truncation of Interface Binding Sites of Crotoxin-A Precursor to Generate Chaperon Subunit of Rattlesnake Neurotoxins}

For all the neurotoxic PLA enzymes the enzymatic activity has been known to be a necessary condition for the neurotoxicity (Yang, 1997; Kattah et al., 2002; Chen et al., 2004). Recently, it has been shown that the product of the PLA reaction have equivalent effects of snake PLA 2 neurotoxins (Rigoni et al., 2005). However, for different venom neurotoxic PLAs catalytic powers and toxicities are not well correlated. Detailed mechanisms and the roles of ester-hydrolysis remain to be solved for neurotoxins, which result in the blockade of neurotransmitter exocytosis.

Crotalid venom evolved neurotoxic PLAs either in monomeric form (e.g., Agkistrodotoxin from Gloydius brevicaudus 
1. S. c. tergeminus (Sistruxin A)

2. c. s. scutulatus (Majava toxin A)

3. c. d. terrificus (Crotoxin A)

4. C. d. cascavella (Subunit A)
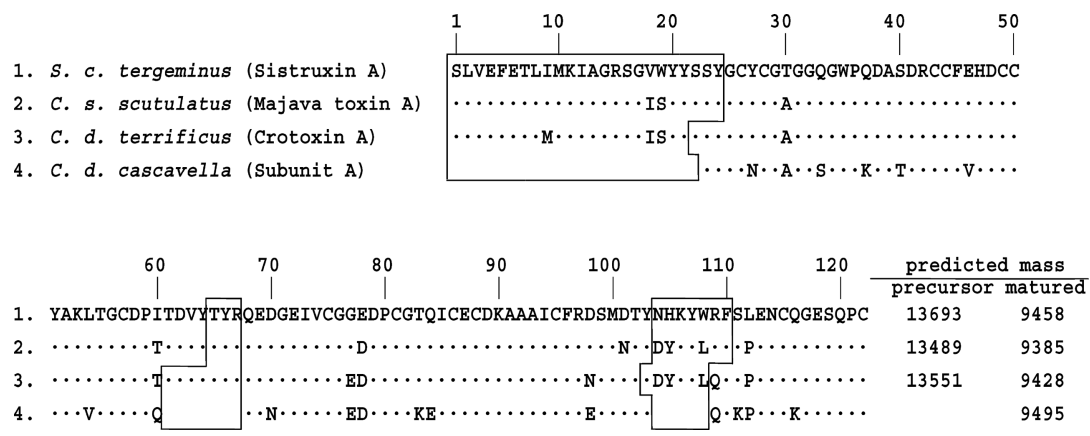

FIGURE 3 Amino acid sequence alignment of crotoxin A-like subunits from Crotalus and Sistrurus venoms. Single-letter codes of amino acids are used, and residues identical to those in the top line are denoted with dots. Three regions, which are removed by proteolysis during the post-translational processing are blocked.

venom) or in heterodimeric form (e.g., crotoxin). Crotoxin-like toxins are present only in venom of neurotoxic rattlesnakes, which possibly evolved from Asian Gloydius (Chen et al., 2004). Expression of a crotoxin A (i.e., crotapotin) gene is a venom marker of the neurotoxic Crotalus and Sistrurus (Wooldridge et al., 2001). The N-terminal region of PLA has been known to be essential for maintaining the active site $\mathrm{H}$-bond network and its interfacial binding (Han et al., 1997; Qin et al., 2005). Thus, specific deletion of three interfacial peptides at 1-23, 69-76, and 113-121 in the precursor by limited proteolysis generated an inactive crotapotin (Fig. 3). The two subunits (crotoxin A and B) form a strong complex and free subunits could hardly be found in the venom (Chen et al., 2004). Binding of crotoxin A increases the toxicity of crotoxin $\mathrm{B}$ by up to 36 -fold, depending on the neuromuscular system assayed (Hermandez-Oliveira et al., 2005), but it inhibits enzymatic activity of crotoxin B in vitro (Chen et al., 2004). Also, crotoxin A itself may have some specific functions on some target cells, e.g. T-lymphocytes (Garcia et al., 2003).

\section{Multiple Mutations of Interfacial Binding Sites in the Acidic PLA-Subunit of Heterodimeric Viperinae Toxins}

Some Viperinae venom species (e.g., those under the genera Daboia and Vipera) contain heterodimeric PLA-toxins (Wang et al., 1992; Francis et al., 1995; Komori et al., 1996; Tsai et al., 
1.RV.7

2. Vipoxin $\operatorname{lnh}$. NLFOEGEMILEKTG-KEVVHSYAIYGCYCGWGGQGRAQDATDRCCFVHDCCYGTVN---DC

3. Vaspin-A

4. P. fiedi Cb-la

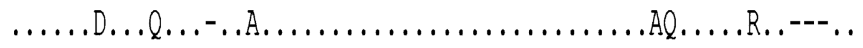

5. V. pales. $\mathrm{VPa}$

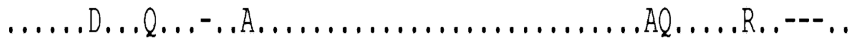
6. Am-11a


H.T...D..NK ...TEGLL..VY .....L..K.KP .............--G. H.S...D..NK...-IFGIM. . IY ........K.KPL............R..---G.

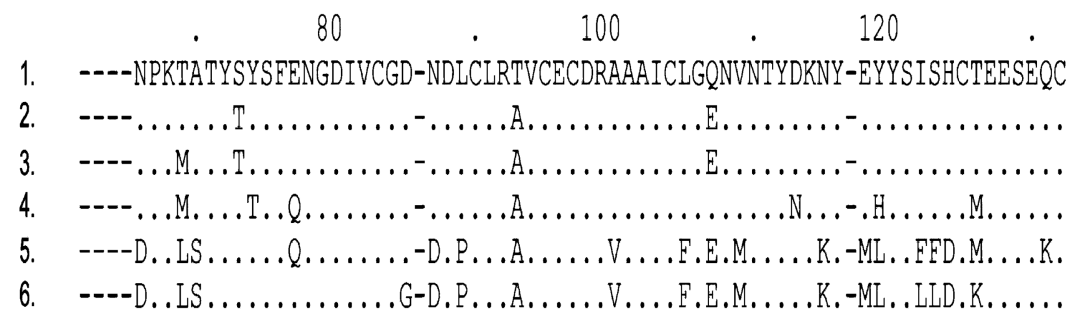

FIGURE 4 Sequence alignments for the acidic subunits of Viperinae heterodimeric PLA toxins (1-4) and two monomeric PLAs (5 and 6). Single-letter codes of amino acids are used, and residues identical to those in the top line are denoted with dots. The numbering system follows that of Renetseder et al. (1985). GenBank or Swiss-prot accession numbers and venom species for the PLAs are: Daboia russelii RV-7, X68386; Vipera ammodytes meridionalis, Vipoxin Inhibitor, P04084; Vipera a. aspis Vaspin-A, AF548351, Am-I1a, AY159807; Pseudocerastes fieldi Cb-Ia (Francis et al., 1995); Vipera palaestinae VPa (Krizag et al., 1996).

1996; Jan et al., 2002). Two PLA subunits designated as RV-4 (pI 10) and RV-7 (pI 4.3) form heterodimers in the venom of Taiwanese Daboia siamensis (Wang et al., 1992; Tsai et al., 1996). RV-7 had very low enzymatic activity (100-300 fold lower than that of RV-4), but it enhanced the neurotoxic and hypotensive effects of RV-4. The amino acid sequences of RV-7 like subunits of different venom species were aligned as in Fig. 4 along with two acidic Viperinae PLAs, which apparently are not a chaperon of basic subunits (Krizaj et al., 1996; Guillemin et al., 2003). Vipoxin and its inhibitor (from Bulgarian V. a. meridionalis venom) share 92\% sequence identities with RV-4 and RV-7, respectively, but vipoxin inhibitor contains His48Gln (Fig. 4) and thus is totally inactivated (Perbandt et al., 2003).

RV-7 also acts like a competitive inhibitor of RV-4 catalyzed hydrolysis in vitro (data not shown). RV-7 had very low enzymatic 
activity (100-300 fold lower than that of RV-4), but it enhanced the neurotoxic and hypotensive effects of RV-4. Its low activities may be attributed to less basic or less hydrophobic substitutions at the interfacial binding sites, i.e. G6, E/D7, E11, E17, Q34, D60, D114, E119, and H124 (Fig. 4). Crystallographic study of the RV-4/RV-7 complex showed that residues N1, Q34, N56, D60, D49 and $\mathrm{K} 69$ of RV-7 are involved in binding to the basicRV-4 subunit (Georgiera et al., 2004). Recently it was found that intravenous injection of RV-7 solution into anesthetized experimental animals (0.1 $\mu \mathrm{g} / \mathrm{g}$ body weight) depressed blood pressure. RV-7 also elicited relaxation in a phenylephrine-precontracted aortic model in vitro, and it was shown that RV-7 might activate eNOS in endothelial cells, and thus relax smooth muscle (Chih, 2001). In addition, oligomeric or complex type presynaptic neurotoxins of Elapid venom species, e.g. taipoxin and textilotoxin, also contain nontruncated, but inactivated PLA chaperon subunits with substitutions at both interfacial binding sites and catalytic sites (Fig. 1). Strong association among subunits of the toxins may reduce non-specific binding and enzyme activity of the only active PLA subunit (Chang, 1985; Tzeng, 1993).

\section{Conclusions}

Besides being hydrolases, secreted PLAs of vertebrates are well known to induce degranulation of mast cells and promote cytokine and chemokine production by direct binding to specific receptors on target cells (Triggiani et al., 2005). So far there is no convincing evidence to support the direct association of venom PLAs with prey-digestion. Many venom PLAs are toxins with specific pharmacological targets but low enzyme activities. During venom evolution, group II basic PLA-cytotoxins may be mutated at the active site Asp49 and loose their essential $\mathrm{Ca}^{+2}$ but retain binding to cell membranes. In contrast, certain Elapid venom basic PLAs are catalytically inactivated by Pro31 substitution. Venom PLAs bind strongly to coagulation factor may alter their interfacial binding sites and no longer hydrolyze the aggregated lipid substrate. To become the chaperon subunits of presynaptic neurotoxins, the acidic PLA may be truncated at the interfacial binding regions or have I-face mutations that allow a strong binding to the basic catalytic active subunits. This 
type of acidic PLAs subunit (e.g., RV7 of Daboia siamensis) itself appears to show other special pharmacological effects. The above examples demonstrate that venom PLAs may play the role of protein scaffold, like the nonenzymatic three-fingered toxins of Elapid venoms and other polypeptide toxins from invertebrate venoms.

\section{Acknowledgments}

This paper is dedicated to late Professor C. C. Yang who has pioneered snake venom research especially the chemical modification of venom phospholipases $A_{2}$. I thank Prof. C. S. Liu for the supply of $B$. fasciatus venom PLAs and advise. I am also grateful to assistants and students in my laboratory during the past 28 years for their efforts, and the continuous support of National Science Council for grant funding, and Mr. S. Y. Tsai for preparation of the Tables and Figures.

\section{References}

Bao, Y., Bu, P., Jin, L., Wang, H., Yang, Q., An, L. (2005). Purification, characterization and gene cloning of a novel phospholipase $\mathrm{A}_{2}$ from the venom of Agkistrodon blomhoffii ussurensis. Int. J. Biochem. Cell Biol. 37:558-565.

Chang, C. C. (1985). Neurotoxins with phospholipase $A_{2}$ activity in snake venoms. Proc Natl. Sci. Counc. Repub. China B. 9:126-142.

Chang, W. C., Lee, M. L., Lo, T. B. (1983). Phospholipase $A_{2}$ activity of longchain cardiotoxins in the venom of the banded krait (Bungarus fasciatus). Toxicon 21:163-165.

Chen, Y. H., Wang, Y. M., Hseu, M. J., Tsai, I. H. (2004). Molecular evolution and structure-function relationships of crotoxin-like and asparagine 6-containing phospholipases $\mathrm{A}_{2}$ in pit viper venoms. Biochem. J. 381:25-34.

Chih, Lan Hui (2001). Pharmacological studies of venom phospholipase $\mathrm{A}_{2}$ isolated from Russell's viper on smooth muscle, National Taiwan University.

Condrea, E., Yang, C.-C., Rosenberg, P. (1982). Additional evidence for a lack of correlation between anticoagulant activity and phospholipid hydrolysis by snake venom phospholipases $A_{2}$. ThromB. Haemost. 47:298-307.

Danse, J. M., Gasparini, S., Menez, A. (1997). Molecular biology of snake venom phospholipases $A_{2}$. In: Kini, R. M., ed. Venom Phospholipase $A_{2}$ Enzyme: Structure, Function and Mechanism. Chichester: Wiley, pp. 29-71.

Francis, B., Bdolah, A., Kaiser, I. I. (1995). Amino acid sequences of a heterodimeric neurotoxin from the venom of the false horned viper (Pseudocerastes fieldi). Toxicon 33:863-874. 
Garcia, F., Toyama, M. H., Castro, F. R., Proenca, P. L., Marangoni, S., Santos, L. M. (2003). Crotapotin induced modification of T-lymphocyte proliferative response through interference with PGE2 synthesis. Toxicon 42:433-437.

Georgieva, D. N., Perbandt, M., Rypniewski, W., Hristov, K., Genov, N., Betzel, C. (2004). The X-ray structure of a snake venom Gln48 phospholipase $\mathrm{A}_{2}$ at 1.9 A resolution reveals anion-binding sites. Biochem. Biophys. Res. Commun. 316:33-38.

Gelb, M. H., Jain, M., Hanel, A. M., Berg, O. G. (1995). Interfacial enzymology of glycerolipid hydrolases: lessons from secreted phospholipases $\mathrm{A}_{2}$. Ann. Rev. Biochem. 64:653-688.

Guillemin, I., Bouchier, C., Garrigues, T., Wisner, A., Choumet, V. (2003). Sequences and structural organization of phospholipase $\mathrm{A}_{2}$ genes from Vipera aspis aspis, V. aspis zinnikeri and Vipera berus berus venom. Identification of the origin of a new viper population based on ammodytin I1 heterogeneity. Eur. J. Biochem. 70:2697-2706.

Gubensek, F., Kordis, D. (1997). Venom phospholipases $A_{2}$ genes and their molecular evolution. In: Kini, R. M., ed. Venom Phospholipase $A_{2}$ Enzyme: Structure, Function and Mechanism. Chichester: Wiley, pp. 73-95.

Han, S. K., Edward, L., Yoon, T., Scott, D. L., Sigler, P. B., Cho, W. (1997). Structural aspects of interfacial adsorption. A crystallographic and site-directed mutagenesis study of the phospholipase $\mathrm{A}_{2}$ from the venom of Agkistrodon piscivorus piscivorus. J. Biol. Chem. 272:3573-3582.

Hernandez-Oliveira, S., Toyama, M. H., Toyama, D. O., Hyslop, S., RodriguesSimioni, L. (2005). Biochemical, pharmacological and structural characterization of a new PLA2 from Crotalus durissus terrificus (South American Rattlesnake) venom. Protein J. 24:233-242.

Inada, M., Crowl, R. M., Bekkers, A. C., Verheij, H., Weiss, J. (1994). Determinants of the inhibitory action of purified 14-kDa phospholipases $\mathrm{A}_{2}$ on cell-free prothrombinase complex. J. Biol. Chem. 269:26338-26343.

Jabeen, T., Singh, N., Singh, R. K., Ethayathulla, A. S., Sharma, S., Srinivasan, A., Singh, T. P. (2005). Crystal structure of a novel phospholipase $A_{2}$ from Naja naja sagittifera with a strong anticoagulant activity. Toxicon 46:865-875.

Jan, V., Maroun, R. C., Robbe-Vincent, A., De Haro, L., Choumet, V. (2002). Toxicity evolution of Vipera aspis aspis venom: identification and molecular modeling of a novel phospholipase $\mathrm{A}_{2}$ heterodimer neurotoxin. FEBS Lett. 527:263-268.

Kattah, L. R., Ferraz, V., Santoro, M., da Silva Camargos, R., Ribeiro, E., Diniz, C., De Lima, M. E. (2002). Analysis of fatty acids released by crotoxin in rat brain synaptosomes. Toxicon 40:43-49.

Krizaj, I., Bieber, A. L., Ritonja, A., Gubensek, F. (1991). The primary structure of ammodytin L, a myotoxic phospholipase $\mathrm{A}_{2}$ homologue from Vipera ammodytes venom. Eur. J. Biochem. 202:1165-1168.

Krizaj, I., Bdolah, A., Gubensek, F., Bencina, P., Pungercar, J. (1996). Protein and cDNA structures of an acidic phospholipase $\mathrm{A}_{2}$, the enzymatic part of an unusual, two-component toxin from Vipera palaestinae. Biochem. Biophys. Res. Commun. 227:374-379. 
Kini, R. M. (2005). Structure-function relationships and mechanism of anticoagulant phospholipase $\mathrm{A}_{2}$ enzymes from snake venoms. Toxicon 45:1147-1161.

Koduri, R. S., Han, S. K., Othman, R., Baker, S. F., Molini, B. J., Wilton, D. C., Gelb, M. H., Cho, W. (1997). Mapping the interfacial binding surface of human secretory group IIa phospholipase A2. Biochemistry 36:14325-14333.

Komori, Y., Masuda, K., Nikai, T., Sugihara, H. (1996). Complete primary structure of the subunits of heterodimeric phospholipase $\mathrm{A}_{2}$ from Vipera a. zinnikeri venom. Arch. Biochem. Biophys. 327:303-307.

Kuipers, O., Kerver, J., van Meersbergen, J., Vis, R., Dijkman, R., Verheij, H. M., de Haas, G. H. (1990). Influence of size and polarity of residue 31 in porcine pancreatic phospholipase $\mathrm{A}_{2}$ on catalytic properties. Protein Eng. 3:599-603.

Landucci, E. C., de Castro, R. C., Toyama, M., Giglio, J. R., Marangoni, S., De Nucci, E. A. (2000). Inflammatory oedema induced by the Lys-49 phospholipase $\mathrm{A}_{2}$ homologue piratoxin-i in the rat and rabbit. Effect of polyanions and p-bromophenacyl bromide. Biochem. Pharmacol. 59:1289-1294.

Lee, W. H., da Silva Giotto, M. T., Marangoni, S., Toyama, M. H., Polikarpov, I., Garratt, R. C. (2001). Structural basis for low catalytic activity in Lys49 phospholipases $\mathrm{A}_{2}$-a hypothesis: the crystal structure of piratoxin II complexed to fatty acid. Biochemistry 40:28-36.

Liu, C. S., Lo, T. B. (1994). Chemical studies of Bungarus fasciatus venom. J. Chinese Biochem. Soc. 23:69-75.

Liu, C. S., Chang, C. S., Leu, H., Chen, S., Lo, T. (1988). The complete aminoacid sequence of a basic phospholipase $\mathrm{A}_{2}$ in the venom of Bungarus fasciatus. Biol. Chem. Hoppe. Seyler. 369:1227-1233.

Liu, C. S., Leu, H. L., Chang, C. S., Chen, S. W., Lo, T. B. (1989). Amino acid sequence of a neutral phospholipase $\mathrm{A}_{2}$ (III) in the venom of Bungarus fasciatus. Int. J. Pept. Protein Res. 34:257-261.

Liu, C. S., Chen, J. M., Chang, C. H., Chen, S. W., Tsai, I. H., Lu, H. S., Lo, T. B. (1990). Revised amino acid sequences of the three major phospholipases $\mathrm{A}_{2}$ from Bungarus fasciatus (banded krait) venom. Toxicon 28:1457-1468.

Liu, C. S., Chen, J. M., Chang, C. H., Chen, S. W., Teng, C. M., Tsai, I. H. (1991). The amino acid sequence and properties of an edema-inducing Lys-49 phospholipase $\mathrm{A}_{2}$ homolog from the venom of Trimeresurus mucrosquamatus. Biochim. Biophys. Acta 1077:362-370.

Liu, C. S., Kuo, P. Y., Chen, J. M., Chen, S. W., Chang, C. H., Tseng, C. C., Tzeng, M. C., Lo, T. B. (1992). Primary structure of an inactive mutant of phospholipase $\mathrm{A}_{2}$ in the venom of Bungarus fasciatus (banded krait). J. Biochem. (Tokyo) 112:707-713.

Lomonte, B., Yamileth, A., Caldero, N. L. (2003). An overview of lysine-49 phospholipase $\mathrm{A}_{2}$ myotoxins from crotalid snake venoms and their structural determinants of myotoxic action. Toxicon 42:885-901.

Magnusson, A. O., Rotticci-Mulder, J. C., Santagostino, A., Hult, K. (2005). Creating space for large secondary alcohols by rational redesign of Candida antarctica lipase B. Chembiochem. 6:1051-1056. 
Mora, R., Maldonado, A., Valverde, B., Gutiérre, J. M. (2006). Calcium plays a key role in the effects induced by a snake venom Lys 49 phospholipase $\mathrm{A}_{2}$ homologue on a lymphoblastoid cell line. Toxicon 46:75-86.

Mounier, C. M., Luchetta, P., Lecut, C., Koduri, R. S., Faure, G., Lambeau, G., Valentin, E., Singer, A., Ghomashchi, F., Beguin, S., Gelb, M. H., Bon, C. (2000). Basic residues of human group IIA phospholipase $A_{2}$ are important for binding to factor $\mathrm{Xa}$ and prothrombinase inhibition comparison with other mammalian secreted phospholipases A. Eur. J. Biochem. 267:4960-4969.

Murakami, M. T., Gabdoulkhakov, A., Genov, N., Cintra, A. C. O., Betzel, C., Arni, R. K. (2006). Insights into metal ion binding in phospholipases $\mathrm{A}_{2}$ : ultra high-resolution crystal structures of an acidic phospholipase $\mathrm{A}_{2}$ in the $\mathrm{Ca}^{2+}$ free and bound states Biochimie. 88:543-549.

Nishida, M., Terashima, M., Tamiya, N. (1985). Amino acid sequences of phospholipases A, from the venom of an Australian elapid snake (king brown snake, Pseudechis australis. Toxicon 23:87-104.

Pan, Y. H., Yu, B. Z., Berg, O. G., Jain, M. K., Bahnson, B. J. (2002). Crystal structure of phospholipase $\mathrm{A}_{2}$ complex with the hydrolysis products of platelet activating factor: Equilibrium binding of fatty acid and lysophospholipidether at the active site may be mutually exclusive. Biochemistry 41:1479014800.

Perbandt, M., Tsai, I. H., Banumathi, S., Rajashankar, K. R., Georgieva, D., Kalkura, N., Singh, T. P., Genove, N., Betzel, Ch. (2003). Structure of the heterodimeric neurotoxic complex viperotoxin F (RV4/RV7) from the venom of Vipera russelli formosensis at 1.9 A resolution. Acta Cryst. D59:16791687.

Polgar, J., Magnenat, E. M., Peitsch, M. C., Wells, T. N., Clemetson, K. J. (1996). Asp-49 is not an absolute prerequisite for the enzymic activity of low-M(r) phospholipases $\mathrm{A}_{2}$ : purification, characterization and computer modelling of an enzymically active Ser- 49 phospholipase $A_{2}$, ecarpholin $S$, from the venom of Echis carinatus sochureki (saw-scaled viper). Biochem. J. 319:961-968.

Prijatelj, P., Charnay, M., Ivanovski, G., Jenko, Z., Pungerčar, J., Križaj, I., Faure, G. (2006). The C-terminal and $\beta$-wing regions of ammodytoxin A, a neurotoxic phospholipase $\mathrm{A}_{2}$ from Vipera ammodytes ammodytes, are critical for binding to factor Xa and for anticoagulant effect. Biochimie 88:69-76.

Qin, S., Pande, A. H., Nemec, K. N., He, X., Tatulian, S. A. (2005). Evidence for the regulatory role of the N-terminal helix of secretory phospholipase $\mathrm{A}_{2}$ from studies on native and chimeric proteins. J. Biol. Chem. 280:36773-36883.

Qi, Y. H., Gong, H., Wieland, S. J., Fletcher, J. E., Conner, G. E., Jiang, M. S. (1989). Effect of a phospholipase $A_{2}$ with cardiotoxin-like properties, from Bungarus fasciatus snake venom, on calcium-modulated potassium currents. Toxicon 27:1339-1349.

Renetseder, R., Brunin, S., Dijkstra, B. W., Drenth, J., Sigler, P. B. (1985). A comparison of the crystal structures of phospholipase $\mathrm{A}_{2}$ from bovine pancreas and Crotales atrox venom. J. Biol. Chem. 260:11627-11634.

Rigoni, M., Caccin, P., Gschmeissner, S., Koster, G., Postle, A. D., Rossetto, S. G., Montecucco, C. (2005). Neuroscience: Equivalent effects of snake 
phospholipase $\mathrm{A}_{2}$ neurotoxins and lysophospholipid -fatty acid mixtures. Science 310:1678-1680.

Rosenberg, P. (1997). Pitfalls to avoid in the study of correlations between enzymatic activity and pharmacological properties of phospholipases $\mathrm{A}_{2}$ enzymes. In: Kini, R. M., ed. Venom Phospholipase $A_{2}$ Enzymes: Structure, Function and Mechanism. Chichester: Wiley, pp. 155-184.

Rojnuckarin, P., Muanpasitporn, C. C., Arpijuntarangkoon, L. J., Intragumtornchai, T. (2006). Molecular cloning of novel serine proteases and phospholipases $\mathrm{A}_{2}$ from green pit viper (Trimeresurus albolabris) venom gland cDNA library. Toxicon. 47:279-287.

Schmidt, J. J., Middlebrook, J. L. (1989). Purification, sequencing and characterization of pseudexin phospholipases $\mathrm{A}_{2}$ from Pseudechis porphyriacus (Australian red-bellied black snake). Toxicon. 27:805-818.

Scott, D. L. (1997). Phospholipase $A_{2}$ : structure and catalytic properties. In: Kini, R. M., ed. Venom Phospholipase $A_{2}$ Enzymes: Structure, Function and Mechanism. Chichester: Wiley, pp. 97-128.

Six, D. A., Dennis, E. A. (200). The expanding superfamily of phospholipase $\mathrm{A}_{2}$ enzymes: classification and characterization. Biochim. Biophys. Acta 1488: $1-19$.

ShiauLin, S. Y., Huang, M. C., Lee, C. Y. (1975). A study of cardiotoxic principles from the venom of Bungarus fasciatus (Schneider). Toxicon. 13:189-192.

Singha, G., Gourinatha, S., Sarvanana, K., Sharmaa, S., Bhanumathib, S., Betzel, Ch., Yadava, S., Srinivasana, A., Singh, T. P. (2005). Crystal structure of a carbohydrate induced homodimer of phospholipase $\mathrm{A}_{2}$ from Bungarus caeruleus at 2.1 A resolution. J. Struct. Biol. 149:264-272.

Takasaki, C., Kimura, S., Kokubun, Y., Tamiya, N. (1988). Isolation, properties and amino acid sequences of a phospholipase $\mathrm{A}_{2}$ and its homologue without activity from the venom of a sea snake, Laticauda colubrina, from the Solomon Islands. Biochem. J. 253:869-875.

Takasaki, C., Yutani, F., Kajiyashiki, T. (1990). Amino acid sequences of eight phospholipases $\mathrm{A}_{2}$ from the venom of Australian king brown snake, Pseudechis australis. Toxicon. 28:329-339.

Thuren, T., Tulkki, A. -P., Virtanen, J. A., Kinnunen, P. K. (1987). Triggering of the activity of phospholipase $\mathrm{A}_{2}$ by an electric field. Biochemistry 26:49074910.

Thunnissen, M. M., Franken, P. A., de Haas, G. H., Drenth, J., Kalk, K. H., Verheij, H. M., Dijkstra, B. W. (1992). Site-directed mutagenesis and X-ray crystallography of two phospholipase $\mathrm{A}_{2}$ mutants: Y52 F and Y73 F. Protein Eng. 5:597-603.

Triggiani, M., Granata, F., Giannattasio, G., Marone, G. (2005). Secretory phospholipases $\mathrm{A}_{2}$ in inflammatory and allergic diseases: Not just enzymes. J. Allergy Clin. Immunol. 116:1000-1006.

Tsai, I. H., Tsai, H. Y., Saha, Y., Gomes, A. (2007). Sequences, geographic variations and molecular phylogeny of venom phospholipases and three fingers toxins of eastern India Bungarus fasciatus and kinetic analyses of its Pro31 phospholipases A2. FEBS J. 274:512-525. 
Tsai, I. H., Chen, Y. H., Wang, Y. M. (2004). Comparative proteomics and subtyping of venom phospholipases $\mathrm{A}_{2}$ and disintegrins of Protobothrops pit vipers. BBA-Prot. and Proteom. 1702:111-119.

Tsai, I. H., Chen, Y. H., Wang, Y. M., Tu, M. C., Tu, A. T. (2001). Purification, sequencing and phylogenetic analyses of novel Lys-49 Phospholipases $\mathrm{A}_{2}$ from the venoms of rattlesnakes and other pit vipers. Arch. Biochem. Biophys 394:236-244.

Tsai, I. H., Wang, Y. M., Au, L. C., Ko, T. P., Chen, Y. H., Chu, Y. F. (2000). Phospholipases $\mathrm{A}_{2}$ from Calloselasma rhodostoma venom gland: cloning and sequencing of ten of the cDNAs, three-dimensional-modelling and chemical modification of the major isozyme. Eur. J. Biochem. 267:6684-6691.

Tsai, I. H., Lu, P. Y., Su, Y. C. (1996). Two types of Russell's viper revealed by variation in phospholipases $A_{2}$ from venom of the sub-species. Toxicon. 34:99-109.

Tsai, I. H., Wang, Y. M., Chen, Y. H., Tsai, T. S., Tu, M. C. (2004). Venom phospholipases $\mathrm{A}_{2}$ of bamboo viper (Trimeresurus stejnegeri): molecular characterization, geographic variations and evidence of multiple ancestries. Biochem. J. 377:215-223.

Tzeng, M. C. (1993). Interaction of presynaptically toxic phospholipase $A_{2}$ with membrane receptors and other binding sites. J. Toxicol. 12:1-62.

Van den Berg, B., Tessari, M., Boelens, R., Dijkman, R., de Haas, G. H., Kaptein, R., Verheij, H. M. (1995a). NMR structures of phospholipase $A_{2}$ reveal conformational changes during interfacial activation. Nat. Struct. Biol. 2:402-406.

Van Den Berg, B., Tessari, M., Boelens, R., Dijkman, R., Kaptein, R., de Haas, G. H., Verheij, H. M. (1995b). Solution structure of porcine pancreatic phospholipase $\mathrm{A}_{2}$ complexed with micelles and a competitive inhibitor. $J$. Biomol. NMR 5:110-121.

Wang, Y. M., Pong, H. F., Tsai, I. H. (2005). Unusual phospholipases $A_{2}$ in the venom of two primitive tree viper Trimeresurus puniceus and Trimeresurus boneenesis. Eur. J. Biochem. 272:3015-3025.

Wang, Y. M., Wang, J. H., Pan, F. M., Tsai, I. H. (1996). Lys-49 phospholipase A $_{2}$ homologs from venoms of Deinagkistrodon acutus and T. mucrosquamatus have identical protein sequence. Toxicon. 34:485-489.

Wang, Y. M., Liew, Y. F., Chang, K. Y., Tsai, I. H. (1999). Purification and characterization of the venom phospholipases $A_{2}$ from four monotypic Crotalinae snakes. J. Nat. Toxins 8:331-340.

Wang, Y. M., Lu, P. J., Ho, C. L., Tsai, I. H. (1992). Characterization and molecular cloning of neurotoxic phospholipases from Taiwan viper (Vipera russelli formosensis). Eur. J. Biochem. 209:635-641.

Ward, R. J., de Azevedo Jr, W. F., Arni, R. K. (1998). At the interface: crystal structures of phospholipases A2. Toxicon. 36:1623-1633.

White, S. P., Scott, D. L., Otwinowski, Z., Gelb, M. H., Sigler, P. B. (1990). Crystal structure of cobra-venom phospholipase $\mathrm{A}_{2}$ in a complex with a transition-state analogue. Science 250:1560-1563.

Wei, J. F., Wei, X. L., Chen, Q. Y., Huang, T., Qiao, L. Y., Wang, W. Y., Xiong, Y. L., He, S. H. (2006). N49 phospholipase $\mathrm{A}_{2}$, a unique subgroup of snake venom group II phospholipase $\mathrm{A}_{2}$. Biochim. Biophys. Acta 1760:462-477. 
Wooldridge, B. J., Pineda, G., Banuelas-Ornelas, J. J., Dagda, R. K., Gasanov, S. E., Rael, E. D., Lieb, C. S. (2001). Mojave rattlesnakes (Crotalus scutulatus scutulatus) lacking the acidic subunit DNA sequence lack Mojave toxin in their venom. Comp. Biochem. Physiol. 130B:169-179.

$\mathrm{Xu}, \mathrm{K}$. (1986). Membrane active polypeptides from venom of Bungarus fasciatus. Biomed. Res. 7(suppl.):89-93.

Yang, C. C. (1997). Chemical modification and fuctional sites of phospholipases $\mathrm{A}_{2}$. In: Kini, R. M., ed. Venom Phospholipase $A_{2}$ Enzymes: Structure, Function and Mechanism. Chichester: Wiley, pp. 155-184.

Yu, B. Z., Berg, O. G., Jain, M. K. (1993). The divalent cation is obligatory for the binding of ligands to the catalytic site of secreted phospholipase $\mathrm{A}_{2}$. Biochemistry 32:6485-6492.

Yu, B. Z., Janssen, M. J., Verheij, H. M., Jain, M. K. (2000). Control of the chemical step by leucine-31 of pancreatic phospholipase $\mathrm{A}_{2}$. Biochemistry 39:5702-5711.

Zhong, X., Jiao, H., Fan, L., Zhou, Y. (2002). Functional important residues for the anticoagulant activity of a basic phospholipases $\mathrm{A}_{2}$ from the Agkistrodon halys Pallas. Prot. Peptide Lett. 9:427-434.

Zuliani, J. P., Fernandes, C. M., Zamuner, S. R., Gutierrez, J. M., Teixeira, C. F. (2005). Inflammatory events induced by Lys-49 and Asp-49 phospholipases $\mathrm{A}_{2}$ isolated from Bothrops asper snake venom: role of catalytic activity. Toxicon. 45:335-346. 